Ann. Biol. anim. Bioch. Biophys., I974, 14 (4-B), 829-835.

\title{
EFFET HYPERMAGNÉSÉMIANT DE QUELQUES PROSTAGLANDINES CHEZ LE RAT
}

\author{
P. LARVOR et Y. RAYSSIGUIER \\ Station de Physiopathologie de la Nutrition, \\ Centre de Recherches zootechniques et vétérinaires, I. N.R. A., \\ Theix, 63110 Beaumont
}

\section{RÉSUMÉ}

Une injection intrapéritonéale de $1 \mathrm{mg} / \mathrm{kg}$ de prostaglandines $\mathrm{PGE}_{1}$ ou $\mathrm{PGE}_{2}$ provoque en une heure une hypermagnésémie significative chez le rat. La calcémie des animaux reste normale. $\mathrm{La} \mathrm{PGF}_{2 \alpha}$ est sans effet sur $\mathrm{Mg}$ ou Ca.

L'hypermagnésémie due aux PGE par voie intrapéritonéale est maximale au bout de I heure, elle est potentialisée par un bloqueur $\alpha$ adrénergique (phentolamine) et nécessite la présence de la médullo-surrénale. Ces caractéristiques la rapprochent beaucoup de l'hypermagnésémie provoquée par la pilocarpine, dont elle ne se distingue que par l'effet d'un bloqueur $\beta$ adrénergique (propranolol). Celui-ci inhibe l'hypermagnésémie par la pilocarpine sans modifier l'hypermagnésémie par les PGE.

\section{INTRODUCTION}

TASHJIAN et al. (I972) ont récemment décrit un effet ostéolytique des prostaglandines sur une culture d'os de souris; il était donc logique de rechercher si une injection de ces hormones était susceptible de modifier la calcémie ou la magnésémie.

\section{MATÉRIEL E'T MÉTHODES}

Nous avons utilisé des rats des deux sexes, de souche Sherman. Les prostaglandines PGE $_{1}$ $\mathrm{PGE}_{2}$ et $\mathrm{PGF}_{2 \alpha}$ ) ont été d'abord dissoutes, dans de l'éthanol pur, puis la solution ramenée à $20 \mathrm{p}$. Ioo d'éthanol environ avant l'injection. L'injection était généralement faite par voie intrapéritonéale, parfois par voie intraveineuse (veine saphène interne). Les témoins recevaient la même quantité de solvant. Les bloqueurs adrénergiques étaient administrés une demi-heure avant l'administration de prostaglandines. Le sang était prélevé par ponction cardiaque, sous 
légère anesthésie à l'êther, dans un tube hépariné. Le plasma était recueilli après centrifugation immédiate, et dosé pour $\mathrm{Mg}$ et $\mathrm{Ca}$ par absorption atomique (Spectrophotomètre d'absorption atomique Perkin-Elmer 303). On a dans certains cas dosé l'acétate sanguin par chromatographie en phase gazeuse selon REMESY et DEMIGNe (I974).

Sur certains animaux, la démédullation surrénalienne a été réalisée selon la technique de INGLE et GRIFFITH (1963).

\section{RÉSULTATS E'T DISCUSSION}

\section{I. - Effet de différentes $P G$ sur la magnésémie du Rat}

Après une heure, l'injection intrapéritonéale de $\mathrm{PGE}_{1}, \mathrm{PGE}_{2}$ ou $\mathrm{PGF}_{2}$ à la dose de $\mathrm{I} \mathrm{mg} / \mathrm{kg}$ de poids vif chez des rats $q$ de $\mathrm{I}_{5} \mathrm{O} \mathrm{g}$, entraîne les modifications de la magnésémie et l'acétate sanguins rapportées dans le tableau I. La magnésémie est significativement élevée par l'injection de $\mathrm{PGE}_{1}$ ou $\mathrm{PGE}_{2}$, tandis que l'acétate sanguin s'abaisse, traduisant l'effet antilipolytique des PGE (BERGSTRÖM et al., I964), la $\mathrm{PGF}_{2 \alpha}$ restant inactive dans les deux cas.

\section{TABLEAU I}

Effet de trois prostaglandines à la dose de $1 \mathrm{mg} / \mathrm{kg}$ de poids vif en injection intraveineuse sur le magnésium et l'acétate sanguin du Rat, après une heure $(\bar{x} \pm \sigma / \sqrt{\mathrm{N}}$, un astérisque pour $\mathrm{P}<0,05$, deux, pour $\mathbf{P}<0,01$, trois pour $\mathrm{P}<0,00 \mathrm{I}$ ). (Nombre d'animaux).

Blood plasma magnesium and acetic acid one hour after $P G$ injection $(\mathrm{rmg} / \mathrm{kg})$. Mean $\perp \mathrm{S}$. E. M. One, two, three asteriks mean repsectively $\mathrm{P}<0,05, \mathrm{P}<0,01, \mathrm{P}<0$, oor. (Number of animals).

\begin{tabular}{|c|c|c|c|}
\hline & & $\begin{array}{c}\mathrm{Mg} \\
(\mathrm{Mg} / 100 \mathrm{ml})\end{array}$ & $\begin{array}{c}\text { Acétate } \\
(\mu \mathrm{M} / 100 \mathrm{ml})\end{array}$ \\
\hline \multirow{4}{*}{ 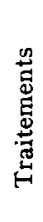 } & Témoins & $1,25 \pm 0,02(8)$ & $22,05 \pm 1,55$ \\
\hline & $\mathrm{PGE}_{1}$ & $1,41 \pm 0,03^{* *}(8)$ & $15,75 \pm 1,34^{* *}$ \\
\hline & $\mathrm{PGE}_{2}$ & $1,42 \pm 0,05^{* *}(8)$ & $12,75 \pm 1,21^{* * *}$ \\
\hline & $\mathrm{PGF}_{2} \alpha$ & $1,26 \pm 0,02(8)$ & $21,25 \pm 1,51$ \\
\hline
\end{tabular}

D'autres essais non rapportés nous ont montré que ce même effet peut être obtenu par injection intraveineuse et qu'il est sensiblement identique chez des rats $\delta$.

\section{2. - Relation dose-réponse entre $P G E_{1}$ et la magnésémie}

Chez des rats $q$ de $\mathrm{I}_{5} \mathrm{o} \mathrm{g}$ des doses progressivement croissantes de $\mathrm{PGE}_{1}$ ont été administrées par voie intrapéritonéale (tabl. 2). L'hypermagnésémie a été significative à partir de $0,5 \mathrm{mg}$ de $\mathrm{PGF}_{1} / \mathrm{kg}$ de poids vif. 
TABI,EAU 2

Effet de différentes doses de PGE sur la magnésémie du Rat après une heure Blood plasma magnesium one hour after increasing doses of $P G E_{1}$

\begin{tabular}{|c|c|c|c|c|c|}
\hline & \multicolumn{5}{|c|}{ Dose de $\mathrm{PGE}_{\mathbf{1}}(\mathrm{mg} / \mathrm{kg})$} \\
\hline & 0 & 0,125 & 0,25 & 0,5 & 1 \\
\hline $\begin{array}{l}\text { Magnésémie } \\
(\mathrm{mg} / 100 \mathrm{ml})\end{array}$ & $1,40 \underset{(8)}{ \pm} 0,03$ & $1,47 \underset{(6)}{ \pm} 0,0^{\prime}$ & $1,49 \underset{(6)}{ \pm} 0,04$ & $1,57 \underset{(6)}{ \pm} 0,03^{* *}$ & $1,67 \pm 0,07^{* *}$ \\
\hline
\end{tabular}

\section{3. - Évolution en fonction du temps de la magnésémie} après injection de prostaglandines

L'injection intrapéritonéale de $\mathrm{PGE}_{1}, \mathrm{PGE}_{2}$ et $\mathrm{PGF}_{2 \alpha}$ à la dose de $\mathrm{I} \mathrm{mg} / \mathrm{kg}$ chez des rats ô de $\mathrm{I}_{5}$ o $\mathrm{g}$ a été suivie d'une prise de sang réalisée à des temps variables après l'injection (fig. I) chaque prise de sang étant réalisée une fois seulement sur un lot de 6 animaux. On voit que la magnésémie maximale se situe environ une heure après l'injection pour les $\mathrm{PGE} ; \mathrm{PGF}_{2 \alpha}$ n'a eu aucun effet sur ce paramètre.

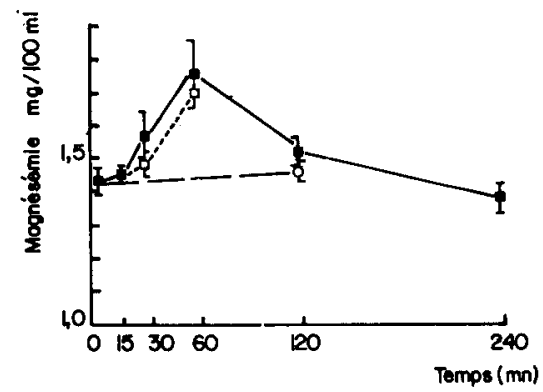

FIG. 1. - Évolution en fonction du temps de la magnésémie du rat après injection de $1 \mathrm{mg} / \mathrm{kg}$ de prosiaglandines

Moyenne \pm erreur type. $T=$ témoins (o), $\mathrm{PGE}_{1}(\mathbf{}), \mathrm{PGE}_{2}(\square)$.

Chaque point représente la moyenne de 6 rats

Plasma magnesium in rats sacrified at different times after administration of $P G E_{1}$ or $P G E_{2}$

Each value represents the mean of 6 rats. Controls (o), $\mathrm{PGE}_{1}(\boldsymbol{\square}) \mathrm{PGE}_{2}$ (ם)

\section{4. - Effet des bloqueurs adrénergiques $\alpha$ et $\beta$ et de la démédullation surrénalienne sur l'hypermagnésémie provoquée par $P G E_{1}$}

Les caractéristiques de l'hypermagnésémie due aux PGE (intensité, délai d'apparition) étant voisines de celles obtenues par une injection de pilocarpine (RAYSSTGUIER et LARVOR, I972 ; et données non publiées), nous avons étudié 1'effet de différentes modifications du système sympathique (bloqueurs $\alpha$ et $\beta$, démédul- 
lation surrénalienne) sur l'hypermagnésémie provoquée par les PGE afin de voir si le système neurovégétatif pouvait être également impliqué dans cette réaction.

Le tableau 3 montre que le propranolol (bloqueur $\beta$ ) ne modifie pas l'effet de la $\mathrm{PGE}_{1}$, tandis que la phentolamine (bloqueur $\alpha$ ) potentialise 1'hypermagnésémie due à cette hormone.

\section{TABLEAU 3}

Effet d'un bloqueur $\alpha$ (Phentolamine) ou $\beta$ (Propranolol) sur l'hypermagnésémie due à $P G E_{1}$

(Mêmes abréviations que tableaux $\mathrm{I}$ et 2 )

Effect of previous injection of phentolamine $(5 \mathrm{mg} / \mathrm{kg})$ or propranolol (10 $\mathrm{mg} / \mathrm{kg}$ ) on hypermagnesemia after $P G E_{1}$

\begin{tabular}{|c|c|c|c|c|}
\hline & & \multicolumn{2}{|c|}{ Dose de $\mathrm{PGE}_{1}$} & \multirow{2}{*}{$\begin{array}{c}\text { Signification } \\
\text { de la } \\
\text { différence } \\
\text { 0-1 mg PGE }\end{array}$} \\
\hline & & 0 & $1 \mathrm{mg} / \mathrm{kg}$ & \\
\hline \multirow{3}{*}{ 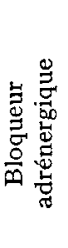 } & 0 & $1,40 \pm \frac{ \pm}{(6)} 0,04$ & $1,68 \underset{(6)}{ \pm} 0,05$ & $* *$ \\
\hline & $\begin{array}{c}\alpha \text { Phentolamine } \\
(5 \mathrm{mg} / \mathrm{kg})\end{array}$ & $1,43 \underset{(6)}{ \pm} 0,02$ & $1,97 \underset{(6)}{ \pm} 0,10$ & $* *$ \\
\hline & $\begin{array}{c}\beta \text { Propranolol } \\
(10 \mathrm{mg} / \mathrm{kg})\end{array}$ & $1,38 \underset{(6)}{ \pm} 0,02$ & $1,69 \pm 0,01$ & ** \\
\hline \multicolumn{2}{|c|}{$\begin{array}{l}\text { Signification de la } \\
\text { différence } 0(\alpha \text { ou } \beta)\end{array}$} & $\begin{array}{l}\alpha \text { NS } \\
\beta \text { NS }\end{array}$ & $\stackrel{* *}{\text { NS }}$ & \\
\hline
\end{tabular}

TABLEAU 4

\section{Effet de la démédullation surrénalienne}

sur la magnésémie du Rat après injection de $1 \mathrm{mg} / \mathrm{kg}$ de $P G E_{1}$, intrapéritonéal (mêmes abréviations que tableaux I et 2 )

Magnesemia after PGE injection and effect of adrenal demedullation

\begin{tabular}{|c|c|c|c|c|}
\hline & & Opératic & réalable & \\
\hline & & Pseudo opérés & Démédullés & \\
\hline & 0 & $1,42 \underset{(8)}{ \pm} 0,03$ & $1,41 \underset{(8)}{ \pm} 0,06$ & NS \\
\hline $\begin{array}{l}\text { Injection } \\
\text { de } \mathrm{PGE}_{1}\end{array}$ & $1 \mathrm{mg} / \mathrm{kg}$ & $1,70 \underset{(10)}{ \pm} 0,04$ & $1,45 \underset{(8)}{ \pm} 0,03$ & $* * *$ \\
\hline & & $* * *$ & NS & \\
\hline
\end{tabular}


Le tableau 4 montre que la démédullation surrénalienne empêche l'hypermagnésémie due à la $\mathrm{PGE}_{1}$ d'apparaître.

Il y a donc une similitude étroite dans le mode d'action sur la magnésémie entre pilocarpine et $\mathrm{PGE}_{1}$, à l'exception de l'effet du propranolol, qui est inhibiteur pour la pilocarpine, mais non pour $\mathrm{PGE}_{1}$.

\section{5. -- Relation dose réponse entre $P G E_{1}$ et magnésémie, avec ou sans potentialisation par la phentolamine}

La figure 2 montre que la courbe dose-réponse PGE -hypermagnésémie a exactement la même pente en présence ou en l'absence de phentolamine. Celle-ci a donc un véritable effet potentialisateur et permet à la dose utilisée $(5 \mathrm{mg} / \mathrm{kg})$ de diviser par un facteur de $\mathrm{I} 2$ la quantité de $\mathrm{PGE}_{1}$ nécessaire pour obtenir un certain effet hypermagnésémiant.

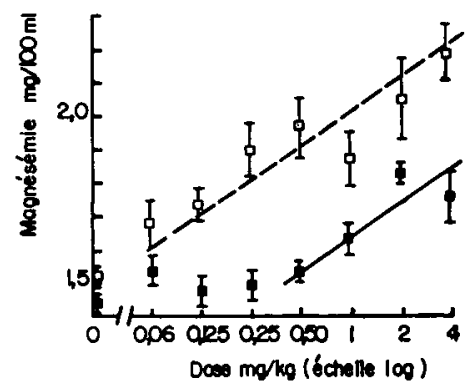

FIG. 2, - Courbe log dose-réponse de l'effet de la PGE sur la magnésémie du rat, sans potentialisation ( $(\mathrm{)}$ ) ou avec potentialisation par la phentolamine ( $\square$ )

Moyenne \pm erreur type. Chaque valeur représente la moyenne de 6 rats

Effect of increasing doses of $P G E_{1}$ on plasma magnesium concentration in rats ( $\square$ ) and effect of previous injection of phentolamine (ם)

Each value represents the mean of 6 rats

\section{DISCUSSION GÉNÉRALE ET CONCLUSIONS}

Il apparait donc une similitude remarquable entre le mode d'action sur la magnésémie du rat d'une injection de pilocarpine ou d'une injection de $\mathrm{PGE}_{1}$ ou $\mathrm{PGE}_{2}$ : les deux nécessitent la présence de la médullosurrénale et sont potentialisés par un bloqueur $\alpha$ adrénergique. La différence essentielle est qu'un bloqueur $\beta$ adrénergique inhibe l'effet de la pilocarpine sans modifier celui des PGE.

Ce parallélisme dans l'action peut être relevé sur différents aspects de l'effet de ces substances sur le système sympathique : il y a hypersécrétion de catécholamines surrénaliennes aussi bien par perfusion de la glande avec la pilocarpine (Douglas et PoIsner, 1965) que par la PGE $_{1}$ (KAYAal,P et TürkER, I967, I968) ; 1a $\mathrm{PGE}_{2}$ a également une action complexe sur les neurones adrénergiques centraux (BERGSTRÖM et al., I973).

Au niveau tissulaire, la sécrétion de noradrénaline par les terminaisons nerveuses est localement inhibée par les PGE (STJÄRNE, 1973), mais cette inhibition peut être 
levée par un bloqueur $\alpha$ adrénergique (STJ ÄRNE, I973), ce qui n'est peut être pas étranger à l'effet de potentialisation de la phentolamine sur l'hypermagnésémie. $\mathrm{Au}$ cours de ces diverses expériences, les PGE, $\mathrm{PGE}_{2}$ ou $\mathrm{PGF}_{2 \alpha}$ n'ont eu aucun effet significatif sur la calcémie, ce qui est en accord avec BEIIEI, et al. (I973) en dépit de l'effet ostéolytique de $\mathrm{PGE}_{2}$ sur l'os en culture ('TAsHJIAN et al., I972).

Ręu pour publication en juin 1974.

\title{
REMERCIEMENTS
}

Nous remercions vivement le Dr J. E. PIke (The Upjohn Company, Kalamazoo, Michigan, U. S. A.) de nous avoir fourni gracieusement les prostaglandines utilisées au cours de ces expériences.

\section{SUMMARY}

\author{
THE EFFECT OF PROSTAGLANDIN INJECTIONS \\ ON PLASMA MAGNESIUM LEVEL, IN THE RAT
}

Prostaglandins (PG) have been shown to stimulate bone resorption (TASHJIAN et al., 1972). We have studied possible changes in plasma Mg concentration after PG injections in rats. Sherman rats of both sexes were used; they were given PG intraperitoneally or vehicle alone (dilute ethanol). Blood was collected by cardiac puncture. Plasma $\mathrm{Mg}$ and $\mathrm{Ca}$ were estimated by atomic absorption spectrophotometry and acetic acid by gas chromatography (REMESY, 1974). PGE 1 or $\mathrm{PGE}_{2}$ ( $1 \mathrm{mg} / \mathrm{kg}$ ) induced hypermagnesemia, no changes in calcemia, and a drop in acetic acid (table I). PGF PG $_{\gamma \alpha}$ had no significant effect on plasma MG, Ca and acetic acid levels. Response to increasing doses of $\mathrm{PGE}_{1}$ and time course of the hypermagnesemic effect were investigated. A significant response could be obtained with $0.5 \mathrm{mg} / \mathrm{kg} \mathrm{PGE}$ (table 2) and the highest plasma magnesium level was observed one hour after injection (fig. I). It has been shown that pilocarpine injection induces hypermagnesemia by stimulating the adrenal medulla and that this effect is inhibited by propranolol and potentialized by phentolamine (RAYSSIGUIER and LARVOR, I972). The effect of PG on magnesemia was not affected by propranolol, but potentiated by phentolamine (fig. 2) and abolished by adrenalde medullation (table 4). It is concluded that hypermagnesemia observed after PG or pilocarpine injection must be considered in relation to the ability of these drugs to increase medullo-adrenal secretion (KAYAALP and TürkER, 1967, 1968).

\section{RÉFÉRENCES BIBLIOGRAPHIQUES}

Bergström S., Carlson L. A., Orö L., r964. Effect of prostaglandins on catecholamine induced changes in the free fatty acids of plasma and in blood pressure in the dog. Acta Physiol. Scand., 60, I70-I 80 .

Bergström S., Farnebo L. O., Fuxe K., I973. Effect of prostaglandin $E_{2}$ on centraland peripheral catecholamine neurons. Eur. J. Pharmacol., 21, 362-368.

Beliel O. M., Singer F. R., Coburn J. W., I973. Prostaglandins : effect on plasma calcium concentration. Prostaglandins, 3, 237-24I.

Douglas W.W., Poisner A. M., I965. Preferential release of adrenaline from the adrenal medulla by muscarine and pilocarpine. Nature, 208, I I02-II03.

INGLE D. M., GRIFFITH J. Q., I963. Surgery of the Rat, in : The rat in laboratory investigation, I vol. $2^{e}$ édition, p. 434-45 I. Farris E. J. et Griffith J. Q., editors, Publishers: Hafner Publ. Co. New York. 
KayaAlp S. O., TürkeR R. K., 1967. Release of catecholamines from the adrenal medulla by prostaglandin $\mathrm{E}_{1}$. Eur. J. Pharmacol., 2, 175-180.

KayaAlP S. O., Türker R. K., r968. Effect of hemicholinium $\left(\mathrm{HC}_{3}\right)$ on the catecholamine releasing action of prostaglandin $\mathrm{E}_{1}$. Eur. J. Pharmacol., 3, 139-I42.

Rayssiguier Y., LARvor P., 1972. Hypermagnésémie et sécrétion médullo-surrénalienne. Ann. Biol. Anim. Bioch. Biophys., 12, 479-491.

Remesy C., Demigne C., 1974. Determination of volatile fatty acids in plasma after ettranolic extraction. Biochem. J., 141, 85-91.

STJÄRNE L., 1973. Dual alpha-adrenoceptor mediated control of secretion of sympathetic neurotransmitter : one mechanism dependent and one independent of prostaglandin E. Prostaglandins, ; $\mathbf{3}$, III-II6.

Tashjian A. H. Jr, Voelkel E. F., Levine L., Goldhaber P., I972. Evidence that the bone resorption-stimulating factor produced by inouse fibrosarcoma cells is prostaglandin $\mathrm{E}_{2}$. $J$. Exp. Med., 136, I329-1343. 